\title{
PHOTOMETRY OF BETELGEUSE FROM THE NATIONAL OBSERVATORY, NAGARKOT, NEPAL
}

\author{
R. K. Koju*, B. Aryal* and S. R. Shahi** \\ *Central Department of Physics, Tribhuvan University, Kirtipur, Kathmandu, Nepal. \\ **B.P. Koirala Memorial Planetarium, Observatory and Science Museum Development Board.
}

\begin{abstract}
Analyzing the visual flux collected by 16 inch Meade LX200GPS telescope placed at National Observatory, Nagarkot, Nepal in FITS format, we noticed a significant asymmetrical feature along the southern region of red giant Betelgeuse. The symmetrical feature is deviated widely when moving from inner to outer layer particularly in the southern direction. The southern region is expanded outward while the northern region is compressed. The region lying in the other two direction remains in intermediate.
\end{abstract}

Keywords: Betelgeuse; Schmidt telescope; Red giant.

\section{INTRODUCTION:}

Betelgeuse (HD 39801, á Orionis) is a red supergiant (RSG) of spectral type M2Iab with magnitude $\langle\mathrm{V}\rangle=0.75$ and color index $\langle\mathrm{B}-\mathrm{V}\rangle=1.86$ located at $05^{\mathrm{h}} 55^{\mathrm{m}} 10.9^{\mathrm{s}},+07^{\mathrm{i}} 24^{\prime} 25.3^{\prime \prime}$ in the Orion constellation. ${ }^{[1]}$ Michelson and Pease (1921) reported its diameter of 0.047 arcsecond at optical wavelength. ${ }^{[2]}$ Since then it has been measured from time to time by a number of interferometers at various wavelengths, giving values that vary substantially with wavelength. Infrared Spatial Interferometer (ISI) measurements showed that the star has systematically decreased in size by about $15 \%$ over

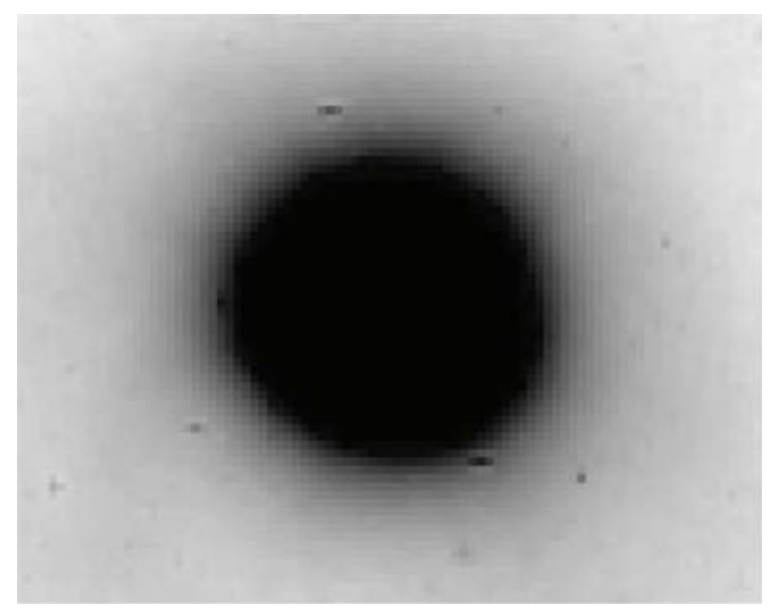

Fig. 1: FITS image of Betelgeuse taken on December 23, 2010 the past 15 years. ${ }^{[3]}$ According to a recent reanalysis of a Hipparcos satellite dataset, its distance is now estimated at $197 \pm 45$ рс. ${ }^{[4]}$ The star rotates with an angular velocity of 1.2 $\mathrm{x} 10^{-8} \mathrm{rad} \mathrm{s}^{-1}$, corresponding to a rotation period of approximately $17 \mathrm{yr}$; the rotation axis has a position angle of $55^{\mathrm{i}}$ from the direction of north in the sky and has an inclination angle of $20^{\circ} .[5]$

Betelgeuse is an enormous giant ranging mass from 15 to $20 \mathrm{M}_{\text {Sun }}$ and is one of the brightest stars visible in the northern winter night sky. Its luminosity is $0.9-1.5 \times 10 \mathrm{~L}_{\text {sun }}$. [6] Very Large Array (VLA) radio interferometry observation revealed an extended, cool ( 1000-3000 K) and irregularly shaped gaseous atmosphere. ${ }^{[7]}$ The extended atmosphere harbors spots, granules and is complex with cool plasma. Kervella et al. (2009) showed vast plumes of gas being ejected into the surrounding atmosphere, possibly containing $\mathrm{CN}$, extending in the southwest direction up to minimum distance of six photospheric radii. ${ }^{[8]}$ Direct Hubble Space Telescope (HST) imagery at ultraviolet wavelength also showed departure of atmosphere from spherical symmetry. ${ }^{\left[{ }^{[9]}\right.}$ Wilson et al. (1997) described optical interferometric observations of Betelgeuse which revealed complex asymmetries in the brightness structure of star with three spots. ${ }^{[10]}$ Thus, we also decided to study its asymmetrical feature by processing FITS image obtained from the National Observatory through the data reduction software ALADIN2.5.

\section{OBSERVATION:}

Betelgeuse was observed through Meade 16 inch LX200GPS telescope placed at the National Observatory,

Author for Correspondence: R. K. Koju, Central Department of Physics, Tribhuvan University, Kirtipur, Kathmandu, Nepal. 


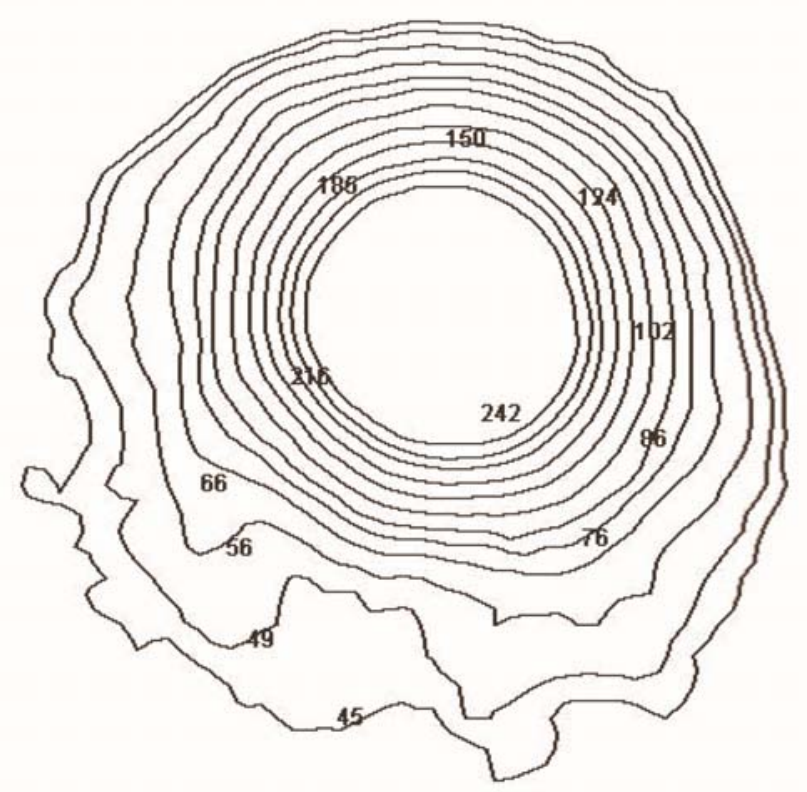

Fig. 2: Different contours are drawn of which contour level'102' in relative unit is chosen as reference for the study of asymmetric feature. Inner contours are symmetrical than that of outer contours. North is to the top and east to the left.

Nagarkot on Dec 23, 2010. Observation was done by tracking an object in two star alignments using the automatic tracking feature in the Autostar II. Photograph of fine and focused image in JPEG and FITS format were taken by replacing eyepiece with webcam through the software AUTOSTAR SUITE3.08.

\section{METHOD OF ANALYSIS:}

Betelgeuse's photographic image in FITS format was processed in the data reduction software ALADIN2.5 to generate information about the value of relative flux density. To study the asymmetrical feature of Betelgeuse various closed contours were drawn of which contour level '102' in relative unit was chosen as reference for the study of asymmetrical feature as contours lying inside it seems to be spherical whereas outside of it appear to be deviating from circular symmetry.

The minor to major axial ratio of contour 102 was found to be 0.93 . A circle having center at the point of intersection of major and minor axis and radius equal to half of minor axis of contour 102 was drawn and decided to study asymmetrical feature in the region lying outside the circle. Spacing between the contours 86-76, 76-66, 66-56, 56-49 and 49-45 were defined as region 1, 2, 3, 4 and 5 . The axes were extended to the outer envelope. We then bisected the image taking major and minor axis as reference.

\section{RESULT:}

Contour mapping on image shows that the contour in the northern direction is closely packed while the contour in the southern direction is widely spaced. This reveals that

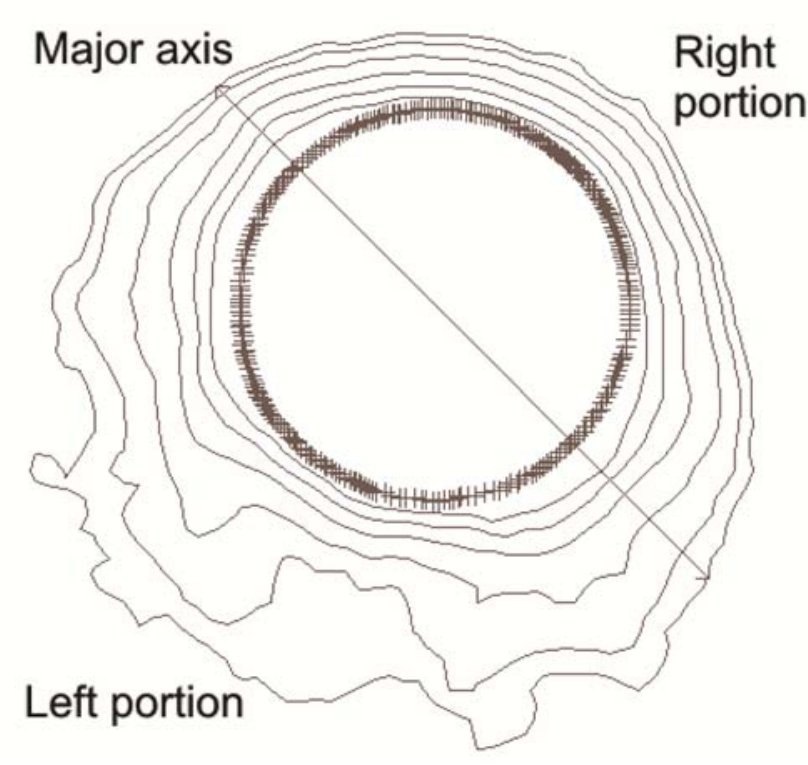

Fig. 3: Image bisection corresponding to major axis

the evolution in the atmosphere of Betelgeuse is taking place more in the southern direction as compared to the other direction giving rise to the asymmetrical feature.

\section{MAJOR AXIS AS REFERENCE:}

Dividing image of Betelgeuse into two parts with respect to major axis as left portion and right portion, the total relative flux density and the number of pixel in the left and the right portion of the outer region 1, 2, 3, 4 and 5 (region is defined section 3 ) are presented in Table 1.

Table 1 Value of relative flux density, pixel number and their ratio when studied the variation of flux density bisecting the star taking reference as major axis. First column lists the region. Next two columns represent the total value of relative flux density and number of pixels. The fourth column gives the average value of flux density. The next three columns are repeated as the $2^{\text {nd }}, 3^{\text {rd }}$ and $4^{\text {th }}$ columns for the right portion.

\begin{tabular}{ccccccc}
\hline \multicolumn{6}{c}{ major axis as reference } \\
\hline \multirow{2}{*}{ region } & \multicolumn{5}{c}{ left portion } & \multicolumn{3}{c}{ right portion } \\
& total flux & total pixel & flux per pixel & total flux & total pixel & flux per pixel \\
\hline I & 22984.94 & 207 & 111.038 & 20101.67 & 181 & 111.059 \\
2 & 30049.55 & 293 & 102.558 & 25783.61 & 250 & 103.134 \\
3 & 54517.10 & 578 & 94.320 & 30969.89 & 327 & 94.709 \\
4 & 84615.00 & 964 & 87.775 & 25888.57 & 295 & 87.758 \\
5 & 80192.00 & 962 & 83.360 & 16765.60 & 201 & 83.411 \\
\hline
\end{tabular}

The difference in the number of pixels and average relative flux density of the left and the right portion is expressed in terms of percentage in Table 2 where $(+)$ sign indicates increase and (-) sign indicates decrease of the left portion with respect to the right portion.

In the innermost region defined by the spacing between the contour $86-76$ as region 1 , the number of pixel in the left portion is $14.36 \%$ more than the right portion. Since all the pixels in the FITS image of Betelgeuse obtained from National 
Table 2: Difference in the number of pixel and flux per pixel of the left portion of major axis with respect to the right portion in terms of percentage. First column lists the region. Second and third column expresses the difference of pixel number and average relative flux density.

\begin{tabular}{ccc}
\hline \multicolumn{2}{c}{ comparison of left with } & respect to right portion \\
\hline region & pixel & flux per pixel \\
\hline 1 & $-10.73 \%$ & $0.042 \%$ \\
2 & $-1.10 \%$ & $0.086 \%$ \\
3 & $-11.06 \%$ & $0.362 \%$ \\
4 & $-40.43 \%$ & $-0.150 \%$ \\
5 & $-48.76 \%$ & $0.222 \%$ \\
\hline
\end{tabular}

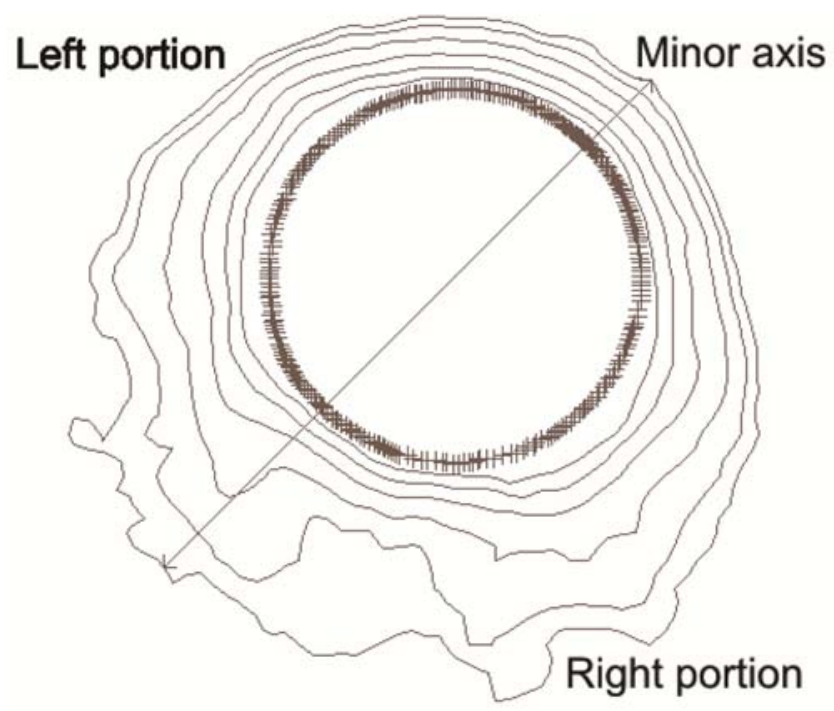

Fig. 4: Image bisection corresponding to minor axis

Observatory are all of the same size, this numerical figure implies that area in the left portion is $14.36 \%$ more than the right portion. The number of pixel of each region in the left portion is greater than the right portion; the value being $17.20 \%, 76.75 \%, 226.78 \%$ and $378.61 \%$ for region $2,3,4$ and 5 respectively. This shows that the symmetrical feature is widely deviated as we proceed towards the outermost envelope. It implies us to deduce that asymmetrical feature exists in the outer atmosphere of Betelgeuse and the outermost envelopes are most important for studying the asymmetrical feature. As all the pixels are of the same size, area of the left portion of the major axis in each region is more than the right portion with the value equal to the corresponding increase in the percentage of the number of pixel.

Though the value of total flux emitted from the left portion in each region is greater than the right portion, the percentage difference in the value of average relative flux density is less than $1 \%$. This indicates that the average energy emitted by each pixel in the left and the right portion are
Table 3: Value of relative flux density, pixel number and their ratio when studied the variation of flux density bisecting the star taking reference as minor axis. First column lists the region. Next two columns represent the total value of relative flux density and number of pixels. The fourth column gives the average value of flux density. The next three columns are repeated as the $2^{\text {nd }}, 3^{\text {rd }}$ and $4^{\text {th }}$ columns for the right portion.

\begin{tabular}{|c|c|c|c|c|c|c|}
\hline \multicolumn{7}{|c|}{ minor axis as reference } \\
\hline \multirow[b]{2}{*}{ region } & \multicolumn{3}{|c|}{ left portion } & \multicolumn{3}{|c|}{ right portion } \\
\hline & total flux & $\begin{array}{l}\text { total } \\
\text { pixel }\end{array}$ & $\begin{array}{c}\text { flux per } \\
\text { pixel }\end{array}$ & total flux & $\begin{array}{l}\text { total } \\
\text { pixel }\end{array}$ & $\begin{array}{l}\text { flux per } \\
\text { pixel }\end{array}$ \\
\hline 1 & 20326.37 & 183 & 111.073 & 22760.24 & 205 & 111.026 \\
\hline 2 & 27774.24 & 270 & 102.868 & 28058.92 & 273 & 102.78 \\
\hline 3 & 40317.15 & 426 & 94.641 & 45169.84 & 479 & 94.3 \\
\hline 4 & 41213.5 & 470 & 87.688 & 69290.07 & 789 & 87.82 \\
\hline 5 & 32895.53 & 394 & 83.491 & 64062.07 & 769 & 83.306 \\
\hline
\end{tabular}

almost equal. The small percentage difference might have result from the unequal distribution in energy during the expansion phase. Betelgeuse is a highly evolved star, one whose central hydrogen fuel supply has run out. The core has contracted into a hot dense state and the outer portions are swollen outward. During this process, inhomogeneous expansion might have taken place producing small variable distribution in energy. The average relative flux density in the left portion is less than the right portion except in region 4.

\section{Minor Axis as Reference}

When the image of Betelgeuse is divided into two parts with respect to the minor axis, the total flux and the number of pixel lying in the left and the right portion of the minor axis are presented in Table 3.

Table 4 shows that number of pixel in the left section is less than the right section in all regions with almost equal value in the region 2 . Thus, when the minor axis is also taken as reference, area in the left section is less than the right section since all the pixels in the image are of the same size. The average relative flux density in the left portion is greater than the right section for the region 1, 2, 3, 5 while it is less for region 4 .

Table 4: Difference in the number of pixel and flux per pixel of the left portion of minor axis with respect to the right portion in terms of percentage. First column lists the region. Second and third column expresses the difference of pixel number and average relative flux density.

\begin{tabular}{ccc}
\multicolumn{2}{c}{ comparison of left with } & respect to right portion \\
\hline region & pixel & flux per pixel \\
\hline 1 & $+14.36 \%$ & $-0.019 \%$ \\
2 & $+17.20 \%$ & $-0.558 \%$ \\
3 & $+76.76 \%$ & $-0.411 \%$ \\
4 & $+226.78 \%$ & $+0.019 \%$ \\
5 & $+378.61 \%$ & $-0.061 \%$ \\
\hline
\end{tabular}




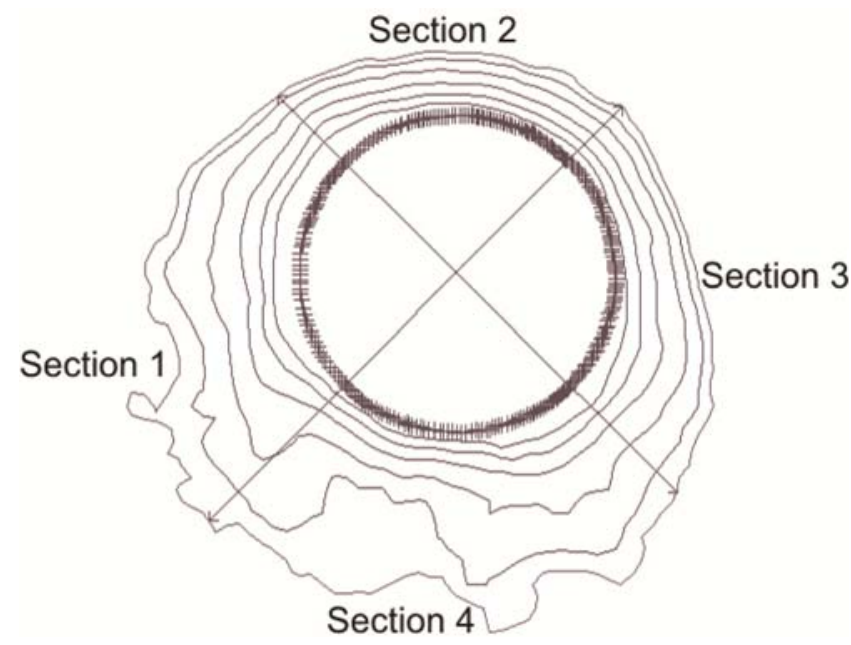

Fig. 5: Divison of image into four parts. Section 2 seems to be more compressed than other sections. North is to the top and east to the left.

\section{Point of Intersection as Reference}

By considering the point of intersection of major and minor axis, image is divided into four sections. The number of pixels lying in these sections is presented in Table 5. It shows that the number of pixel in the section 4 lying in the southern direction is highest and is smallest for the section 2 lying in the northern direction. There exists intermediate value in the number of pixel lying in the east and the west section such that the west section is greater than the east section. Thus, it can be concluded that the atmosphere of

Table 5: Number of pixel in the section formed by the

\begin{tabular}{cc}
\hline section & Total pixel \\
\hline 1 & 1252 \\
2 & 491 \\
3 & 763 \\
4 & 1752 \\
\hline
\end{tabular}

Betelgeuse is asymmetric with the extension much more in the southern direction and the least in the northern direction as the total pixel number only refers to the area of each section.

\section{Flux Density Variation along Major and Minor Axis}

The circle was found to be enclosed between the contour 124 and 86 . Thus, we studied the variation of relative flux density along major and minor axis from the contour 124 to the outermost contour of Betelgeuse. Graph represents the plot of relative flux density and pixel number from the top to the bottom of major and minor axis with the breakdown for the inner region (region lying inside contour 124). The standard error of relative flux density is used as error bar. Graph shows that the relative flux density increases from the peripheral region to the central region (breakdown region) and then decreases to the peripheral region.

The curve fitting of relative flux density against pixel number at the top and the bottom of major and minor axis is presented in Fig. 7 and Fig. 8 where dash line represents linear fit and solid curve represents second order polynomial fit. At the top of major axis, the fitted second order polynomial
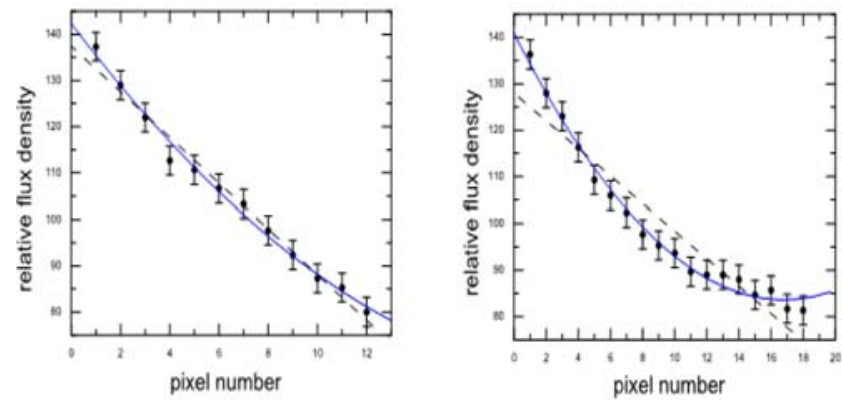

Fig.7: Flux density variation at (left) top and (right) bottom of major axis. The error bar is the standard error of the distribution. Dash and solid curve represents linear fit and second order polynomial fit respectively.

is $y=0.16 x^{2}-7.02 x+142.30$ with error $0.05,0.68,1.94$ respectively in the coefficient and standard deviation of 1.87 while at the bottom of major axis is $y=0.20 x^{2}-6.81 x+$ 140.79 with offset $0.02,0.34$ and 1.40 respectively in the coefficient and standard deviation of 1.76 .

The relative flux density variation along the minor axis also follows the second order nature defined by the equation
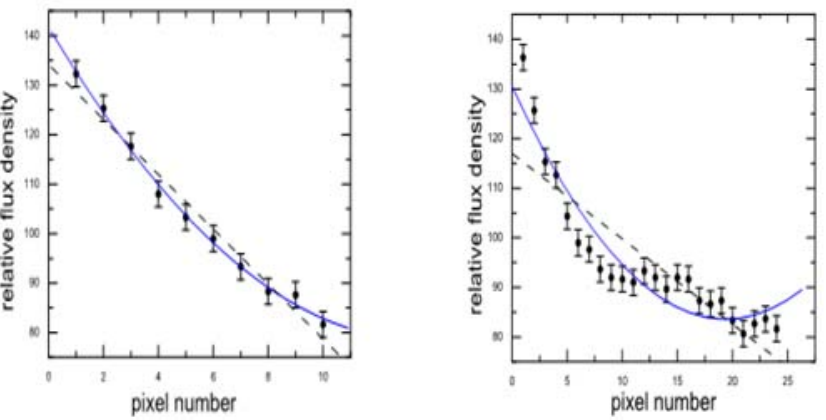

Fig.8: Flux density variation at (left) top and (right) bottom of minor axis. The error bar is the standard error of the distribution. Dash and solid curve represents linear fit and 

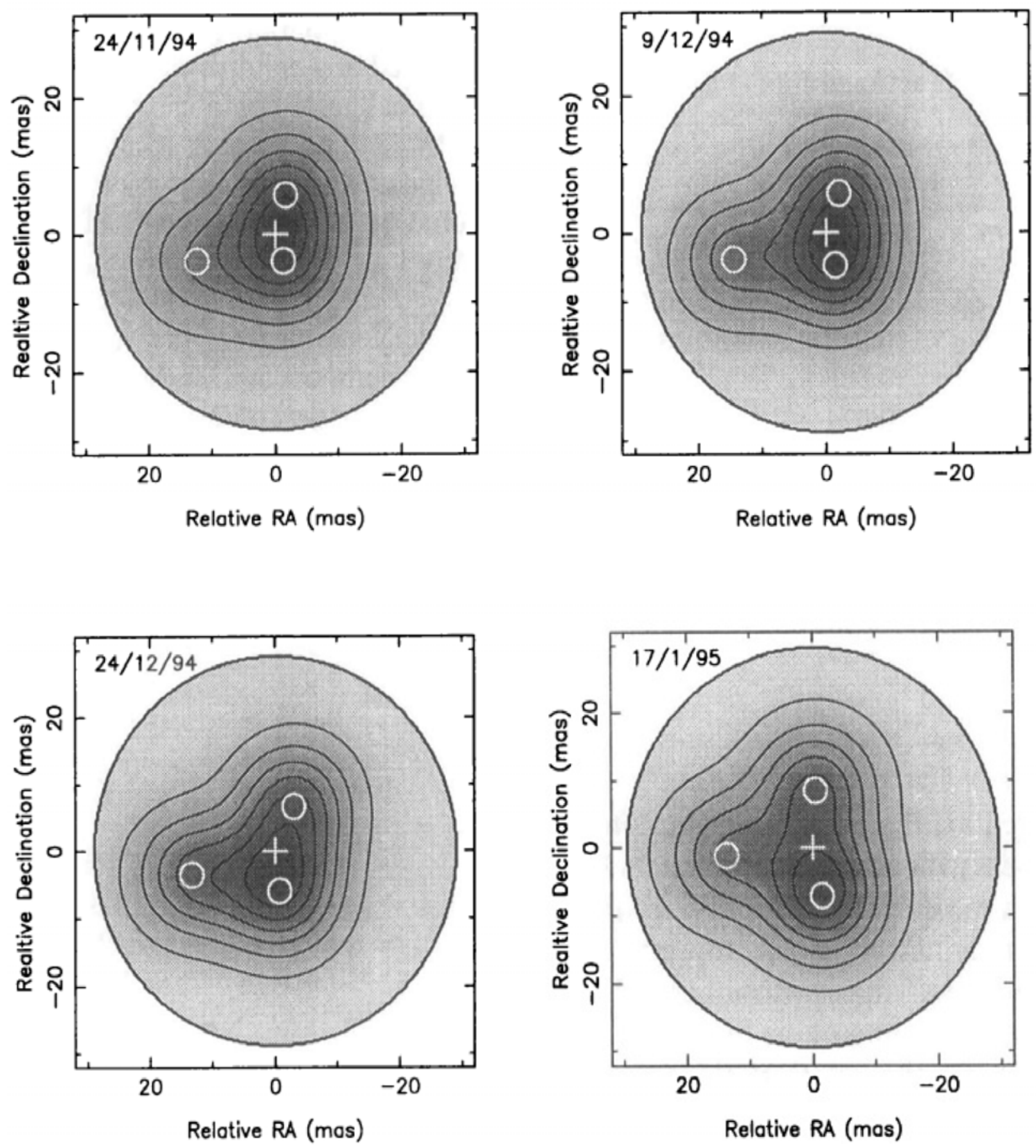

Fig. 9: In Wilson et al. 1997, contours are at $30, \ldots 90$ percent of the peak flux. Small circles locate the center of the spot components on the models and the map center is marked with a plus sign. North is to the top and east to the left. [source: Wilson et al. 1997)

$y=0.34 x^{2}-9.32 x+141.71$ at the top with error $0.06,0.65$, 1.56 in the coefficient and standard deviation of 1.32 while at the bottom with the equation $y=0.13 x^{2}-4.84 x+130.48$ with error in the coefficient $0.02,0.12,3.19$ and standard deviation of 4.79 . Therefore, with the consideration of the variation of relative flux density in these four directions, it can be stated that the flux varies following the second order polynomial in the overall outer atmosphere of Betelgeuse.

\section{DISCUSSION}

Asymmetrical feature exists significantly in the outer envelope of Betelgeuse. Thus, asymmetrical feature should be studied significantly in the outermost envelope of the atmosphere. The atmosphere is much more extended in the southern direction and least in the northern direction with the intermediate value in the eastern and the western direction. The concentration and expansion of the contour 
suggest that there is much more variation in the relative flux density in the northern direction and least in the southern direction with respect to distance from the core. We have also noticed significant broadening along south-east direction. Using non-redundant aperture mask method, Wilson et al. (1997) described optical interferometric observations of Betelgeuse which reveal complex asymmetries in the brightness structure of star with three spots as shown in Fig. 9. They noticed a widening along north-south direction and significant broadening along eastern part in the image taken during 1994-1995. In our study, the image is slightly different with a significant broadening along south-east and the southern direction. We would like to report this change after 15 years that is observed through 16 inch Meade telescope from our National Observatory.

\section{CONCLUSION}

In the visible wavelength, the outer atmosphere of Betelgeuse is outstretched in the southern direction giving rise to asymmetrical feature. We encourage further monitoring of Betelgeuse using the same telescope to follow up this work to obtain concrete evidence about its atmosphere.

\section{ACKNOWLEDGEMENTS}

We acknowledge Central Department of Physics, Tribhuvan University for providing various supports during master thesis. R. K. Koju also acknowledges B.P. Koirala Memorial Planetarium, Observatory and Science Museum and Development Board for financial support to carry out the dissertation work.

\section{REFERENCES}

[1] Krisciunas, K., Information Bulletin on Variable Stars, 2104 (1982).

[2] Michelson, A., Pease, F.G., PNAS, 7, 143 (1921).

[3] Townes, C.H., Wishnow, E.H., Hale, D.D.S., Walp, B. ApJ, 697, L127 (2009)

[4] Harper, G.M., Brown, A., Guinan, E.F., AJ, 135, 1430

[5] Uitenbroek, H., Dupree, A.K., Gilliland, R.L., AJ, 116, 2501 (1998).

[6] Ryde, N., Smith, N., Hinkle, K.H., astro-ph, arxiv: 0811.03037v1 (2008).

[7] Lim, J., Carilli, C.L., White, S.M., Beasley, A.J., Marson, R.G., Nature, 392, 575 (1998).

[8] Kervella, P., et al., arxiv: 0907.12843 (2009).

[9] Gilliland, R.L., Dupree, A.K., ApJ, 463, L29 (1996).

[10] Wilson, R.W., Dhillon, V.S., Hani, C.A., MNRAS, 291, 819 (1997). 